\title{
GALLSTONE DISEASE (CHOLELITHIASIS) - PATHOGENESIS, PREVENTION AND CONTEMPORARY METHODS OF IMAGING DIAGNOSTICS
}

\author{
Bistra Velchovska ${ }^{1}$, Zhenya Zhekova-Maradzhieva ${ }^{1}$, Atanas Uzunov ${ }^{1}$, Evgeniya Ivanova ${ }^{2}$, \\ Desislava Petrova ${ }^{3}$, Mariana Yordanova ${ }^{4}$, Georgi Valchev ${ }^{5}$ \\ ${ }^{1}$ Student, TRS X-Ray Laboratory Assistant, Medical College, Medical University of Varna \\ ${ }^{2}$ Student, TRS Assistant Pharmacist, Yordanka Filaretova Medical College, \\ Medical University - Sofia \\ ${ }^{3}$ Student, TRS Assistant Pharmacist, Medical College, Medical University of Varna \\ ${ }^{4}$ TRS X-Ray Laboratory Assistant, Medical College, Medical University of Varna \\ ${ }^{5}$ Department of Radiology, St. Marina University Hospital, Varna
}

\begin{abstract}
INTRODUCTION: Cholelithiasis is a metabolic disorder, leading to stone formation in the bile ducts and gallbladder. The stones are classified by their composition as cholesterol, pigment, and mixed. The condition is more frequent in overweight individuals, with a stationary way of life, diabetics, and women on oral contraceptives. There is a female predilection with a 3.5:1 ratio. The disease has several forms. The latent one is devoid of complaints - stones are an incidental finding. The acute form manifests with right upper quadrant pain. Biliary colic is typical - it comes in fits of right subcostal pain, nausea, and frequently - vomiting. Complications are frequent - gallbladder and bile duct inflammation, biliary obstruction, gallbladder perforation, and biliary peritonitis.
\end{abstract}

AIM: To analyze the pathogenesis, the prevention and the modern medical imaging methods related to the gallstones disease.

MATERIALS AND METHODS: This research applied statistical methods. The data was processed through statistical and graphical analysis.

RESULTS: Accurate diagnosis requires a compound approach. Anamnesis of biliary colic initiates it. Abdominal ultrasonography is the fastest and most accessible imaging method for finding gallstones. It can also present the gallbladder itself - whether it is enlarged, inflamed, or folded. Additional methods include magnetic resonance imaging (MRI), computed tomography (CT), and some hybrid techniques. If the data of cholelithiasis is ambiguous, the latter can be confirmed by endoscopic retrograde cholangiopancreatography (ERCP) an endoscope is introduced to the level of the papilla of Vater, and contrast is injected into it. The biliary pathways also used to be imaged by percutaneous transhepatic cholangiography, which now is a dated technique.

CONCLUSIONS: Prophylaxis includes avoiding risk factors of alimentary nature and undergoing periodic control ultrasonographies, especially in individuals with a family history of gallstones. This is crucial, as chronic

Address for correspondence:

Bistra Velchovska

TRS X-Ray Laboratory Assistant

Medical College

Medical University of Varna

84 Tsar Osvoboditel Blvd

9000 Varna

e-mail:bistra_bs@yahoo.com cholecystitis, derived from lithiasis, can lead to precancerosis.

Keywords: bile, stone disease, calculi, medical imaging, cholelithiasis, examination 
Bistra Velchovska, Zhenya Zhekova-Maradzhieva, Atanas Uzunov et al.

\section{INTRODUCTION}

Gallstone disease (cholelithiasis) is a disease causing metabolic disruptions of bile acids and cholesterol which result in the formation of stones (concrements) in the bile ducts and the gall bladder.

Gallstone disease is one the most frequent gastroenterological diseases. It affects females in childbearing age more often due to the hormonal changes during pregnancy and as a result of the hormonal and ovarian cycle. The incidence of the disease increases with age, affecting most often female patients between 40 and 60 years of age; in males it is rarer and can be expressed at a later age (3).

The disease is frequent mostly in developed countries in Europe and America. More frequently, it affects overweight patients, women taking hormonal medications (birth control drugs, substitution therapy), women who have given birth, persons with a cholesterol-rich diet, patients with family hyperlipidemia and hypercholesterolemia, patients on a antilipidemic medications treatment, patients with diabetes mellitus, those with myxedema, etc. (3).

Cholelithiasis is characterized by the presence of stones in the gall bladder. Biliary colic is developed due to an obstruction of the biliary passability by a gallstone. Cholesterol gallstones represent $75 \%$ of all stones and the other $25 \%$ are pigment ones. Cholesterol gallstones are associated with overweight, diabetes mellitus (metabolic syndrome) and female patients. Pigmented stones are associated with liver cirrhosis and hemolysis which call for definitive differential diagnosis.

\section{MATERIALS AND METHODS}

This paper summarizes the etiology and the diagnostic methods of this condition in order to increase the awareness of patients and, respectively, to improve their health status. This research applies statistical methods. The data was processed through statistical and graphical analysis.

\section{RESULTS AND DISCUSSION}

Gallstones have a crystal structure and are formed by normal and abnormal bile contents. They can be singular or multiple. The stones can be divided into three groups:

$\diamond$ Cholesterol stones
They represent $10 \%$ of all types of stones. Macroscopically, they have spherical or oval form. Most frequently the stones are singular and their dimensions reach up to $5 \mathrm{~cm}$ of diameter. Their color is white or pale yellow.

$\diamond$ Bilirubin stones

They can be found in 15\% of the patients with cholelithiasis. Macroscopically they contain a certain amount of calcium which is the reason for their characteristic hardness. They are multiple and facetted, with sharp edges. The stones have dark green to green-red color. They reach $10 \mathrm{~mm}$ of diameter.

\section{$\diamond$ Mixed}

They can be found in $75 \%$ of the patients with cholelithiasis. Macroscopically they can be singular but most frequently multiple stones can be observed. They are facetted and rarely exceed $1 \mathrm{~cm}$ in diameter. Their color is greenish, pale-yellowish or black, depending on which of their containing components dominates. Their surface is fine and grainy and they have hard consistency. When cut, a brown-red color covering and a nucleus of light brown radial cholesterol structure can be observed. When cutting, concentric and lamellar structure can be observed. They are comprised of bilirubin, calcium and cholesterol.

The function of the gall bladder is to concentrate and store the bile, and to separate it in the duodenum after the consumption of a meal. The active sodium transport through the gallbladder wall concentrates the bile 10 times above the normal concentration in which it is secreted by the liver. The concentration process leads to a change in the calcium and the cholesterol contained in the bile juice. Cholelithiasis occurs due to a change in the solubility of the gallbladder, which leads to precipitation (delay) of the calcium and cholesterol contained in it (1).

Acute cholecystitis develops due to an obstruction (blockage) of the bile duct, which can be of inflammatory type or purely mechanical, from biliary sludge or gallbladder stone. In approximately $50 \%$ of the patients with acute cholecystitis, there is no evidence of bacterial infection (1).

Choledocholithiasis is characterized by the presence of a stone in the hepatic or the general biliary passage and can result in the development of cholangitis, obstructive jaundice and/or pancreatitis. Cholangitis is defined through the Charcot's triad 
which includes: jaundice, fever and palpable sensitivity in the upper abdominal quadrant. The cholangitis pentad of Reynolds includes the cited 3 symptoms plus hypotonia and disrupted mental status. Cholangitis is an urgent medical condition and requires immediate adequate treatment in order to avoid the development of sepsis (1).

There are a number of risk factors which create a prerequisite for the formation of bile concrements:

$\diamond$ Innate factors - frequent cases of cholesterol stones among certain families and groups;

$\diamond$ Gender - the proportion females to males is 3:1, pregnancy, intake of estrogen medications;

$\diamond$ Age - cholelithiasis becomes more frequent with the advance of age;

$\diamond$ Nutritional factors - cholesterol-rich diet, which lacks fiber; starvation, parenteral nutrition;

$\diamond$ Obesity - cholelithiasis is more common in overweight persons;

$\diamond$ Certain medications;

$\diamond$ Risk factors can be summed up by the so-called 5 -F rule: "female, fair, fat, forty, fertile" (2). The clinical symptoms of the disease can be:

1. Asymptomatic progress of the disease - the condition is discovered randomly by an x-ray examination, scanner or abdominal echography.

2. Dyspeptic form with abnormal symptoms like heaviness in the stomach, bitter taste, abdominal swelling.

3. Painful crisis form - a typical expression, characterized by colic pain in the right upper quadrant irradiating to the back and the right shoulder, nausea, vomiting, and sometimes accompanied by yellowing of the eyes and dark-colored urine. It usually continues from one to four hours, sometimes longer. The crises usually appear after consuming fatty meals, fried food, eggs, cabbage, aubergines, chocolate, etc. The complicated colic may lead to an inflammation of the gall bladder and the bile ducts, perforation of the gall bladder with development of peritonitis, acute pancreatitis and others. The main diagnostic method for gallstone disease is abdominal ultrasound, which allows imaging the number and the volume of the concrements, the volume of the gall bladder, the thickness of its wall, the latter being an important indicator for an inflammation process, as well as the condition of the bile ducts (6).

The imaging diagnostic methods for the condition are:

$\diamond$ Ultrasound examination:

This is the fastest and most accessible way to prove the presence of gallstones which gives the opportunity to avoid more invasive methods, at least at the beginning. It helps prove the presence of an enlarged gall bladder, any changes of its form and walls, obstructed (dilated) ductus choledochus, allows examining its contraction ability after food consumption. Through the ultrasound analysis it cannot be decided whether the diagnosis is cholesterol stones and if they are calcined (9).

$\diamond$ Contrast-enhanced ultrasound-guided percutaneous cholangiography:

The aim of this method is to diagnose the biliary obstruction through the use of a microbubble contrast agent. The used contrast is SonoVue produced by Bracco, $2.5 \mathrm{ml}$, dissolved in $30 \mathrm{ml}$ physiological saline solution, which is applied in slow flow through a preliminarily installed percutaneous transhepatic biliary catheter into the biliary duct system. The examination is conducted though dynamic ultrasound scanning (4).

$\diamond$ Radiological diagnostics:

* Native (dry) image: the calcined stones $(20 \%$ of the cases) are distinguished as a strong shadow; the rest of the stones can be visualized as a defect in filling only after the application of a contrast agent.

* Radiological examination with iodine containing contrast substances.

Due to the high sensitivity of the ultrasound examination and the opportunity to prove with certainty the presence of stones in the choledochus through ERCP, a radiological examination with iodine-containing contrast substance is only conducted if specific indications exist. The perioral application of the contrast substance (perioral cholecystography) is sufficient.

$\diamond$ Computed tomography (CT) of the gall bladder is the most sensitive method to prove the presence of gallstones (9). 
Bistra Velchovska, Zhenya Zhekova-Maradzhieva, Atanas Uzunov et al.

The method is more effective in finding the cause and the level of biliary obstruction. CT can demonstrate the distal choledochus well and establish the lithiasis if the calculi have sufficient calcium density. Computed tomography has a reduced value in assessing the biliary pathology, especially for benign stenosis. The contrast-enhanced computed tomography is not precise for diagnosing lithiasis of the bile ducts as the majority of the concrements are radiopositive and CT does not involve a venous contrast (7).

$\diamond$ Direct cholangiography: ERCP and PTC can demonstrate the biliary tree in the best possible way. The direct cholangiography can intensify the cholangitis - sepsis, so it should always be accompanied by therapeutic drainage when a biliary obstruction is present.

$\diamond$ Endoscopic retrograde cholangiopancreatography (ERCP) is an examination where a small camera, installed on a flexible tube is introduced through the mouth, passes through the esophagus and the stomach and reaches the spot where the general bile duct opens into the duodenum (the initial part of the small intestine). A contrast substance that is visible by an $\mathrm{x}$-ray apparatus can be injected into the general bile duct. The way in which the bile ducts are shown can provide important information about the problem. A biopsy of liver tissue can be taken in order to be examined microscopically for the existence of cancer. During the ERCP, measures against the existing obstruction can be taken (8).

The endoscopic retrograde cholangiopancreatography (ERCP) is already entirely replaced as a diagnostic method by computed tomography and MRI and more specifically by magnetic resonance cholangiopancreatography (MRCP).

$\diamond$ Percutaneous transhepatic cholangiography (PTC) - the contrast substance is injected percutaneously into the liver and the method gives the opportunity:

1. to exclude the existence of malignant tumors in the bile ducts or the periampullary region;

2. to diagnose sclerosing cholangitis;

3. to avoid the loss of time associated with intraoperative cholangiography;
4. to receive better quality radiographies compared to the intraoperative ones.

During the Dormia basket examination, an attempt to remove the stone is performed. If this method does not succeed, the classic surgical intervention is performed.

In cases with dilated choledochus, above $6 \mathrm{~mm}$, and increased values of GGT (Gamma-glutamyltransferase), alkaline phosphatase and bilirubin, the presence of choledocholithiasis is very probable. If the dimensions of the choledochus are up to $6 \mathrm{~mm}$, and the values of the above-cited laboratory tests are normal, choledocholithiasis is less probable. This can be proven with certainty by endoscopic retrograde cholangiography or by magnetic resonance cholangiography, which has recently replaced it (5).

$\diamond$ Magnetic resonance cholangiopancreatography (MRCP)

Quick, non-invasive, highly-sensitive method for assessing the biliary tree pathology. Two types of contrast mediums are used depending on their ability to decrease or to increase the signal during the examination. The images are received before and after the injection of the contrast and the contrast absorption is different for the different nosological units. The differential contrast enhancing aims at discriminating the pathological area (to limit the pathological area) (7).

A large number of patients with various pathologies can be diagnosed precisely through a quick and safe method such as MRCP. MRCP is better than CT in detecting small concrements due to the significantly better technical ability to precisely limit solid and liquid substances. For this method the minimum possible volume of a concrement reaches up to $3 \mathrm{~mm}$. It is very convenient to perform MRCP before ERCP as a diagnostic method offering the opportunity to discuss the further therapeutic endoscopic options (7).

The method is valuable and remains without an alternative for pregnant women, for patients with changed anatomy after a biliodigestive surgery, and for patients with acute pancreatitis. The advantages of this method consist of not using ionized radiation, the excellent diagnostics of soft tissues and its very good dimensional resolution. The disadvantages of this examination are that it is not possible to take ma- 
terial for microscopic examination (biopsy) and it is still the most expensive method. The main contraindications refer to patients who have a pacemaker and also to persons with metal plates in the body. For claustrophobic patients sedation during the examination is necessary (7).

$\diamond$ cholangiography (x-ray examination of the bile ducts) - it can be performed after injecting a coloring agent into the liver. This allows the physician to follow the passage of the bile as it is drained through the liver. This procedure also allows taking tissue for biopsy and removing different obstructions and narrowings.

In cases with non-complicated gallstone disease and the discovery of small cholesterol stones, the diet intended for patients with bile colic or acute cholecystitis is recommended. These conditions can be provoked by certain foods, that is why carbohydrates should be reduced, the consumption of foods rich in cholesterol (brains, animal fat, animal insides) is forbidden. In cases of complicated gallstone disease, the operative removal of the gall bladder is obligatory as disease recurrences can lead to serious complications (sepsis, acute surgical abdomen, gall bladder cancer, etc.) (2).

\section{CONCLUSION}

Cholelithiasis is a chronic disease which is characterized by the formation of concrements in the gall bladder and the bile ducts. The disease is more common in populations consuming more meat and less common among vegetarians. It is almost four times more common in females and mostly among the overweight ones (9).

Prevention consists of avoiding predisposing factors of nutritional and other nature, especially for persons with hereditary predisposition. This is obligatory as cholelithiasis is a precancerous condition for almost $34 \%$ of the diseases after the age of 50 years.

The most important point is that every patient with cholelithiasis should be monitored and regularly consulted by their general practitioner, gastroenterologist and surgeon.

\section{REFERENCES}

1. Cholelithiasis. c2011, March 13 (cited 2016, August).Available from:http://medicine-bg.net/diferentzialna-diagnoza/1205-816 (in Bulgarian) (3)
2. Cholelithiasis. Doctoral website; (cited 2016,August). Available from: http://www.medik. bg/?page $=$ cat\&id=53 (in Bulgarian) (4)

3. Cholelithiasis MKB K 80 (2015, October 20). Retrieved 2016 August, Available from: medpedia.framar.bg/заболявания/мкб-k80-3/k80жлъчнокаменна-болест-холелитиаза (in Bulgarian) (1)

4. Hadzhiev S. Diagnostic and therapeutic percutaneous procedures of some diseases of abdominal and retroperitoneal organs from the gastrointestinal tract; Abstract, c2013 (cited 2016,August). Available from: http://www.vma.bg/doc/proceduri/doctor/handzhiev/Avtoreferat.pdf (9)

5. Kotzev I. Cholelithiasis. Contemporary perspective, Internet medical journal for doctors in Bulgaria, (2011, issue 11). Available from http://www. medinfo.bg/spisanie/2011/11/statii/jlychnokamenna-bolest-holelitiaza-br-syvremenen-pogled-1207 (11)

6. Penkov V. How to remove gallstones, c2011, February 20 (cited 2016, August). Available from www.marica.bg/как-се-премахват-камъни-вжлъчката-news46555.html (6)

7. Petrov P. Recent researches in the diagnosis of obstructive icterus. 2015. (cited 2016,August). Available from: www.marica.bg/как-се-премахваткамъни-в-жлъчката-news46555.html (10)

8. Petrov Z. Diseases of the biliary tract. 2009 (updated 2010 Okt 24; cited 2016 Aug 25). Available from:http://www.puls.bg/illnes/issue_147/part_5. html (7)

9. Tushev R. Cholelithiasis.Vita Hospital Website. Retrieved 2016, August . Available from http://vita.bg/ статия/жлъчнокаменна-болест-cholelithiasis/50

(5) 